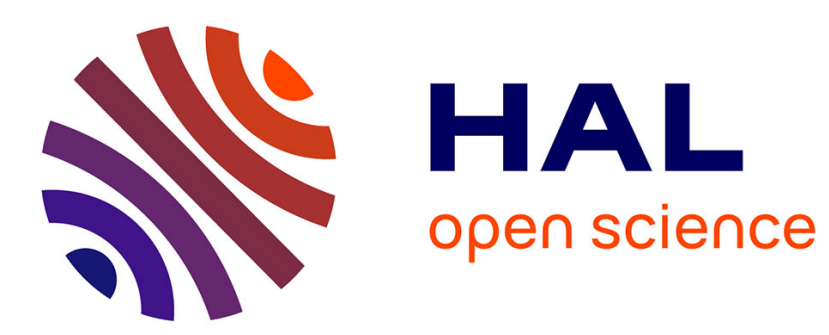

\title{
Effect of Core Impurity on Kink Soliton Motion in Dislocation Line
}

G. Zheng, J. Zhang

\section{To cite this version:}

G. Zheng, J. Zhang. Effect of Core Impurity on Kink Soliton Motion in Dislocation Line. Journal de Physique IV Proceedings, 1996, 06 (C8), pp.C8-277-C8-280. 10.1051/jp4:1996859 . jpa-00254667

\section{HAL Id: jpa-00254667 https://hal.science/jpa-00254667}

Submitted on 1 Jan 1996

HAL is a multi-disciplinary open access archive for the deposit and dissemination of scientific research documents, whether they are published or not. The documents may come from teaching and research institutions in France or abroad, or from public or private research centers.
L'archive ouverte pluridisciplinaire HAL, est destinée au dépôt et à la diffusion de documents scientifiques de niveau recherche, publiés ou non, émanant des établissements d'enseignement et de recherche français ou étrangers, des laboratoires publics ou privés. 
JOURNAL DE PHYSIQUE IV

Colloque C8, supplément au Journal de Physique III, Volume 6, décembre 1996

\title{
Effect of Core Impurity on Kink Soliton Motion in Dislocation Line
}

\author{
G.P. Zheng and J.X. Zhang \\ Department of Physics, Zhongshan University, 135 Xin Gang Road West, 510275 GuangZhou, China
}

\begin{abstract}
Modified Sine-Gordon equation is established to investigate the kink soliton motion affected by core impurities. The dragging force that the core impurities exert on the moving kink soliton is determined by computer simulation of diffusing of solute atoms in dislocation core. The soliton motion can be dissolves into two kinds of movement: the changing of soliton position and soliton shape. These two kinds of movements correspond to the longitude core diffusion(LCD) and transverse core diffusion(TCD) of solute atoms respectively. We solve the equation of these motions numerically, temperature internal friction peak and stress amplitude internal friction peak are discussed. The results are compared with the anomalous interrial friction peaks in $\mathrm{Al}-\mathrm{Mg}, \mathrm{Al}-\mathrm{Cu}$ alloy.
\end{abstract}

\section{Introduction}

The diffusion of solute atoms in alloy, has two kinds of different methods, namely, the bulk diffusion along the dislocation line and the diffusion in the dislocation core. Diffusion of solute atoms in dislocation core has lower activation energy.

In 1950,T.S.Ge[1] had observed a nonlinear anelatic internal friction (NLAIF) peak in Al-Cu alloy, which showed abnormal stress amplitude effect. This phenomena had furtherly studied by $K e$ and his coworkers in Al-Mg,Al-Cu alloy[2], and a serials of nonlinear relaxation peaks were found. At room temperature, the IF peaks were marked as $P_{0}, P^{\prime}{ }_{1}, P^{\prime \prime}{ }_{1}$ from low to high temperature respectively. The activation energy of these IF peaks was approximately one half of it in bulk diffusion, therefore, $\mathrm{P}_{0}, \mathrm{P}_{1}{ }_{1}, \mathrm{P}^{\prime \prime}{ }_{1}$ are considered as nonlinear power dissipations of atoms diffusion in dislocation core.In 1960's, Ke and Zhang[3] proposed a moving kink model to illustrate the abnormal behavior of $\mathrm{P}^{\prime \prime} \uparrow$ peak in Al-Cu alloy. Quantitative calculation of internal friction of moving kink caused by TCD and LCD should be taken based on this model.

Some dislocation internal friction theory had focused on nonlinear anelatic internal friction(NLAIF). For example ,mechanical or thermal pinning (depinning) of dislocation string line from pinors[4], or furthermore, the anchor points in dislocation string line[5]; and diffusion of solute atoms in dislocation core[6][7]. But these models had not considered the kink configuration in dislocation line. As supported by the experiment performed by $\mathrm{Ke}$ et.al, the nonlinearity of moving kink due to the interaction between solute atoms and kink, should be taken into account of NLAIF.

\section{Computer Simulation study on the Dragging Force of Core Impurities.}

The moving dislocation line on the Peierls potential can be treated as one-dimension atomic model developed by Frenkel[8] and Seeger[9].In'order to simulate the dragging effect of the core impurities, we should use the following second-order differential-difference equations to describe the propagating kink in dislocation line

$$
\frac{d^{2} u_{i}}{d t^{2}}+\left(2 u_{i}-u_{i+1}-u_{i-1}\right)+\lambda_{0}^{2} \sin u_{i}=F_{\bar{a}}-f_{d}
$$

Here $\lambda_{0}=(2 \pi / a)\left(U_{0} / \kappa\right)^{1 / 2} ; \kappa$ the the elastic constant of relative atom displacement $; u_{i}$ is the displacment (scaled with respect to $a / 2 \pi$ )of the $i$-th atom in Frenkel model; $f_{d}$ and $F_{a}$ are the dragging force and applied external force respectively(scaled with respect to $2 \pi \kappa / a)$; and $t$ is time scaled with respect to $(\kappa / m)^{1 / 2}$. a is the lattice 
parameter; $m$ and $U_{0}$ are atom mass and Peierls barrier height respectively. we use periodic boundary condition: $u_{0}=u_{n}+2 \pi$.

The core impurities move according to the Langvin equation :

$$
\begin{aligned}
& \frac{d r}{d t}=\frac{D}{T} f_{d^{\prime}}(t)+\eta_{s}(t) \\
& <\eta_{s}(t)>=0,\left\langle\eta_{s}(t) \eta_{s}\left(t^{\prime}\right)>=2 D \delta\left(t-t^{\prime}\right)\right.
\end{aligned}
$$

$r$ is the position vector of the core impurities related to the kink center $X_{c} . D$ the diffusion constant and $T$ the temperature near the dislocation. The dragging force in Eq.(2) can be determined by the following relation[6] :

$$
f_{d}=-\frac{\partial U}{\partial r}, U=\frac{A_{0}}{\left(r+x_{c}\right)^{3}+r_{0}^{3}}
$$

Here $U$ is the interaction energy between impurity and kink and $A_{0}$ the interaction constant. we use Eq.(1) to determined at each time step $\Delta t$ the kink center position $X_{c}$ and kink velocity $V_{C}=d X_{C} / d t$ (scaled with respect to $\left(m / k a^{2}\right)^{1 / 2}$, then the dragging force $f_{d}$ can be calculated from Eq. (2)-(3).

Fig. 1 shows the dragging force at various temperature when the kink moves under constant applied force. The force-velocity relation shown in Fig.(1) can be characterized as the followings:

$$
F_{d}=B_{0} \frac{v_{c} / v_{0}}{1+\left(v_{c} / v_{0}\right)^{2}}
$$

$B_{0}$ is the viscosity coefficient ; $V_{0}=D / a$ is impurity jump velocity. This Debye type dissipation had been proposed to calculate kink dynamics in Ref[6] and Ref[10].

\section{Motion of Kink-soliton under Periodic Applied Stress .}

It is well khown that in the frame of continum approach, we will obtain Sine-Gordon equation (all in dimensionless unit):

$$
\phi_{\xi \xi}-\phi_{\tau \tau}=\sin \phi
$$

This equation has a solution of kink-type soliton: $\phi_{0}(\xi)=4 a r c t g(e \xi)$

it presents the characteristic shape of dislocation kink. (here :

$\left.\phi(x, t)=2 \pi u(x, t) / a ; \quad \zeta=x \omega_{0} / c_{0} ; \quad \tau=\omega_{0} t ; \quad c_{0}=a \sqrt{\frac{k}{m}}, \lambda_{0}=\frac{k^{2}}{2 \pi} \sqrt{\frac{k}{U_{0}}}, \omega_{0}=\frac{c_{0}}{\lambda_{0}}\right)$

Now we exam an impurity situated at $\xi=0$, the interaction potential can be approximated as the following simple form:

$$
g(\xi)=\alpha\left[\theta\left(\xi-\xi_{0}\right)-\theta\left(\xi+\xi_{0}\right)\right]
$$

$\xi_{0}$ is the dislocation core radio $\left.\approx 2 a\right)$. Taking into account the dissipation effect and the pinning effect of the core solute atom, we derive the modified Sine-Gordon equation:

$$
\begin{aligned}
& \phi_{\tau \tau}-\phi_{\xi \xi}+\sin \phi+F\left(\phi_{\xi^{\prime}} \phi_{\tau}, \xi\right)=\sigma a b e^{i \omega \tau}=A e^{i \omega \tau} \\
& F\left(\phi_{\xi^{\prime}} \phi_{\tau}, \xi\right)=B_{0} \frac{\left(\phi_{\tau} / v_{0}\right)}{1+\left(\phi_{\tau} / v_{0}\right)^{2}}+\alpha\left[\delta\left(\xi-\xi_{0}\right)-\delta\left(\xi+\xi_{0}\right)\right]
\end{aligned}
$$

Using perturbation theory. We can expand $\phi$ in the complete set of basic function $f_{k}(\xi)$ described in Ref[11]:

$$
\phi(\xi, \tau)=\phi_{0}(\xi, \tau)+\frac{1}{8} \Psi(0, \tau) f_{0}(\xi)+\int \Psi(k, \tau) f_{k}(\xi) d k
$$

Therefore Eq.(7) can be dissolves into two set of equations:

$$
\Psi_{\tau \tau}(k, \tau)+\Omega^{2}(k) \Psi(k, \tau)+B_{0} \frac{\Psi_{\tau}(k, \tau) / \nu_{0}}{1+\Psi_{\tau}^{2}(k, \tau) / \nu_{0}^{2}}=A \sqrt{\frac{\pi}{2 \Omega(k)}} e^{i \omega \tau} \sinh \left(\frac{\pi k}{2}\right)
$$




$$
\begin{aligned}
& \Psi_{\tau \tau}(0, \tau)+B_{0} \frac{\Psi_{\tau}(0, \tau) / v_{0}}{1+\Psi_{\tau}^{2}(0, \tau) / v_{0}^{2}}+\beta(\xi) \cdot \Psi(0, \tau)=2 \pi A e^{i \omega \tau} \\
& \beta(\xi)=\frac{1}{3} \alpha X_{0}^{2} \exp \left(-\xi_{0}^{2}\right) \cos ^{2}(\Omega \tau), \quad X_{0}=\frac{A}{\Omega^{2}-\omega^{2}}, \quad \Omega^{2}=\frac{\alpha \sinh \xi_{0}}{2 \cosh ^{2} \xi_{0}}
\end{aligned}
$$

Use soliton coordinate frame $(\xi, \tau)$, the change of soliton position relative to its proper frame is :

$$
x_{0}(\tau)=\frac{1}{8} \Psi(0, \tau)
$$

while $\Psi(\xi, \tau)$ correspond to the changing of soliton shape due to the perturbation :

$$
\Psi(\xi, \tau)=\int \Psi(k, \tau) \dot{f}_{k}(\xi) d k
$$

in a dissipation medium, the soliton is a deformed particle under the action of harmonic force. Eq.(9) and Eq.(10) described the deformation affected by transerve diffusion of core impurities(TCD) and longitude diffusion(LCD) respectively.If soliton movement is affected by TCD,Eq.(10) can be ignored because soliton does not displace relative to the soliton coordination frame. If soliton motion was controlled by LCD, (9) should not be considered because of the slight change of the soliton kink shape, the soliton can be treated as a oscillator in the $\xi$-direction under applied force in the soliton coordinate frame.

For summary ,Eq.(9)、Eq. (10) will be used to calculate the internal friction of TCD and LCD; we have to turn to the computer aided numerical calculation. During TCD , the energy dissipation of soliton is determined by:

$$
Q_{T}^{-1}=\frac{G}{\pi A} \int_{-\infty}^{\propto} d \xi \int_{0}^{\omega / 2 \pi} e^{i \omega \tau} \Psi_{\tau}(\xi, \tau) d \tau
$$

In LCD process , the internal friction can be calculated as follow:

$$
Q_{L}^{-1}=\frac{G}{\pi A} \int_{0}^{\omega / 2 \pi} X_{\tau}(0, \tau) e^{i \omega \tau} d \tau
$$

\section{Amplitude Dependent internal Friction Peak Due to LCD and TCD}

The quantity of parameter mentioned in the above equations could be found in Ref[2]. Then we get the numerical result of internal friction peak at various stress amplitude,these curves are shown in Fig. 2 and Fig.3. When stress amplitude increases, the peak temperature of TCD internal friction peak shifts to higher temperature and peak intensity decrease. When the temperature increase, the amplitude internal friction peak appears in high amplitude and IF decrease.

If LCD is dominated, the internal friction peak shifts to lower temperature When $V_{0}$ increase, it has different amplitude behaviours with TCD process.

\section{Discussion and Conclusion}

The dislocation kink can be treated as a soliton, the motion of a kink satisfies the Sine-Gordon equation. When solute atoms diffuse in dislocation core, the damping of the kink result in the changing of soliton position and shape.

We used Debye dissipation term to described the damping of dislocation in solute atom atmosphere in dislocation core. When TCD is dominated, the position of soliton does not change relate to the soliton coordinate frame, but the shape of soliton will be modified by TCD process. The numerical result of internal friction peak and amplitude peak agree with experiment result of $P^{\prime \prime} 1$ peak. When LCD is considered, the shape of soliton was slightly modified. The perturbation of LCD on the motion soliton is to change its position. The motion equation of soliton in this situation interprets the $P_{1}^{\prime}$ peak well.

The condition that core impurities perform their TCD and LCD may be related to their activation over the Peierls barrier of the first kind and the second kind respectively. Further analyse of these process will be given. 


\section{References}

[1]T.S.Ke . Phys.Rev. 78 (1950)420.

[2]T.S.Ke. Progress In Nature Science. 3 (1990)289.(in Chinese)

[3]T2.S.Ke, Z.S.Zhang. Acta. Physica Sinica. 22(1966)71.

[4]K.Lucke , A.V.Granato , J.Teutonico. J.Appl. Phys. 39(1968)5181

[5]W.Winkler-Gniewek. Thesis, Aachen(1973).

[6]Z.Q.Sun , T.S.Ke . Scripta Metal. 15(1981)763.

[7]J.Ni. T.S.Ke. Phys. Stat. Sol.(a). 98(1986)487.

[8]J.T.Frenkel, T.A.Kontorova, Phys. Z. SU13(1938)1.

[9]A.Seeger. J. De. Physique C5. 48(1981)201.

[10]T.Ogurtani, A.Seeger. Phys. Rev. B 31(1985)5044.

[11]M.B.Fogel, S.E.Trullinger, A.R.Bishop, J.A.Krumhansl, Phys. Rev. B 15(1977)1578.
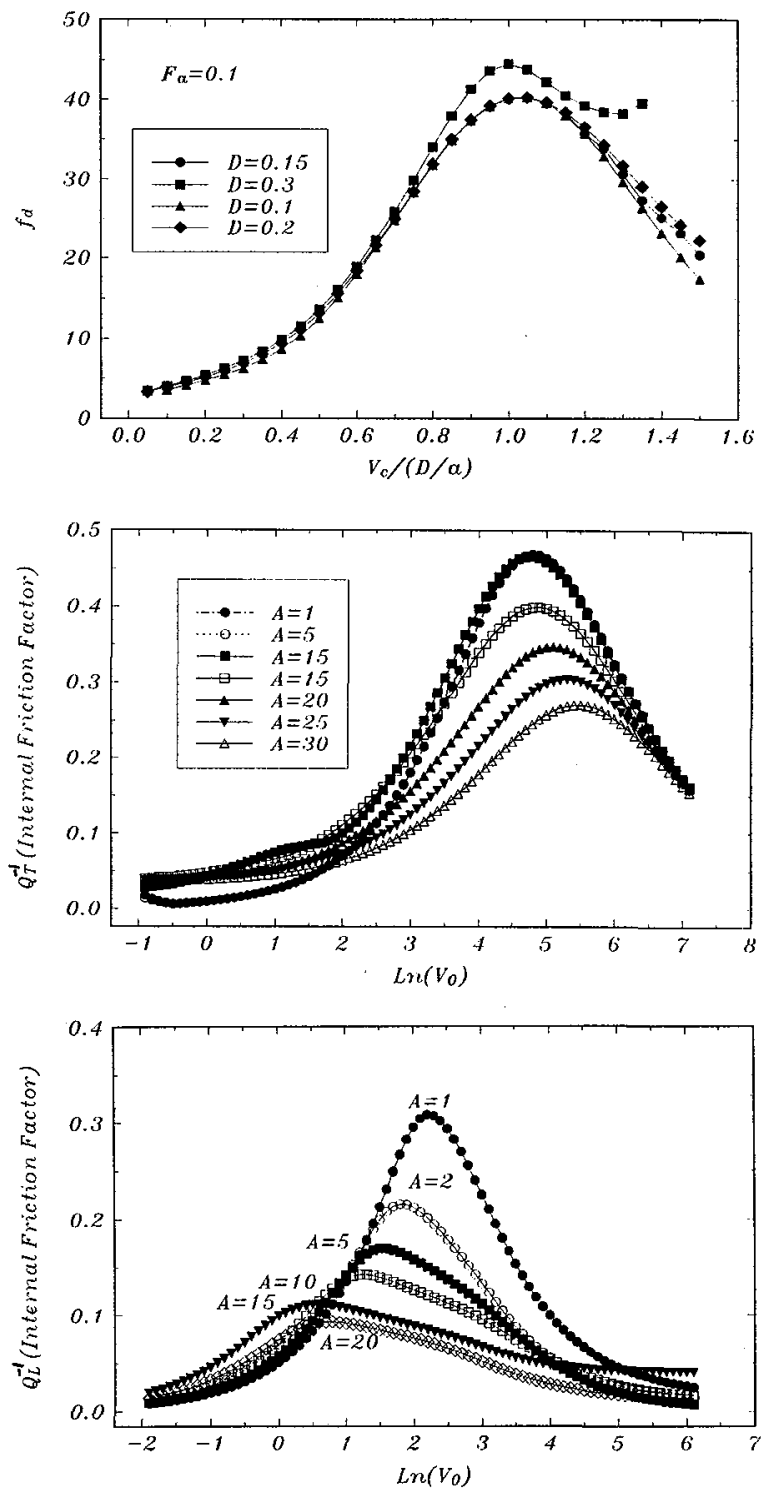

Figure 1: Dragging force of core impurities at different temperature $D=D_{0} \exp \left(-U / k_{B} T\right)$. Atom numbers $\mathrm{N}=500$, kink wide $\mathrm{W}=30$ atom span, $\Delta t=0.001, \hat{\lambda}_{0}=0.5, A_{0} / k_{B} T=2 ; r_{0}=2$.

(in dimensionless unit).

Figure 2: Temperature dependence $Q_{T}^{-1}$ under various stress amplitude $A$. Internal friction is normalized to $G / 2 A$
Figure 3: Temperature dependence $Q_{L}^{-1}$ under various stress amplitude $A$. Internal friction is normalized to $G / 5 A$. 